\title{
Persistence and eventual demise of oxygen molecules at terapascal pressures
}

\author{
Jian Sun, ${ }^{1, \text { f }}$ Miguel Martinez-Canales, ${ }^{2}$ Dennis D. Klug, ${ }^{3}$ Chris J. Pickard, ${ }^{2}$ and Richard J. Needs ${ }^{4}$ \\ ${ }^{1}$ Lehrstuhl für Theoretische Chemie, Ruhr-Universität Bochum, 44780 Bochum, Germany \\ ${ }^{2}$ Department of Physics and Astronomy, University College London, \\ Gower Street, London WC1E 6BT, United Kingdom \\ ${ }^{3}$ Steacie Institute for Molecular Sciences, National Research Council of Canada, Ottawa, K1A 0R6, Canada \\ ${ }^{4}$ Theory of Condensed Matter Group, Cavendish Laboratory, \\ J J Thomson Avenue, Cambridge CB3 OHE, United Kingdom
}

(Dated: September 26, 2021)

\begin{abstract}
Computational searches for structures of solid oxygen under pressures in the multi TPa range have been carried out using density-functional-theory methods. We find that molecular oxygen persists to about $1.9 \mathrm{TPa}$ at which it transforms into a semiconducting square spiral-like polymeric structure $\left(I 4_{1} / \mathrm{acd}\right)$ with a band gap of $\sim 3.0 \mathrm{eV}$. Solid oxygen forms a metallic zig-zag chain-like structure $(\mathrm{Cmcm})$ at about $3.0 \mathrm{TPa}$, but the chains in each layer gradually merge as the pressure is increased and a structure of $F m m m$ symmetry forms at about $9.5 \mathrm{TPa}$ in which each atom has four nearest neighbors. The superconducting properties of molecular oxygen do not vary much with compression, although the structure becomes more symmetric. The electronic properties of oxygen have a complex evolution with pressure, swapping between insulating, semiconducting and metallic.
\end{abstract}

PACS numbers: $61.50 . \mathrm{Ks} 71.20 .-\mathrm{b}$ 81.05.Zx 81.40.Vw

Among the simple molecules studied at high pressures, oxygen has attracted particular attention due to its fundamental importance and intriguing properties. [1 5] For example, oxygen is the only known elemental molecule that exhibits magnetism, which adds substantial complexity to its phase diagram. When liquid oxygen is cooled at ambient pressure it undergoes a sequence of transitions to the $\gamma, \beta$ and $\alpha$ solid phases at $54.39,43.76$ and $23.88 \mathrm{~K}$, respectively [6]. Upon increase of pressure the monoclinic $\alpha-\mathrm{O}_{2}(C 2 / m)$ phase transforms into an orthorhombic $\delta-\mathrm{O}_{2}(F m m m)$ phase at about $3 \mathrm{GPa}$ and to $\epsilon-\mathrm{O}_{2}$ at about $10 \mathrm{GPa}$. The structure of $\epsilon-\mathrm{O}_{2}$ has only recently been solved by x-ray diffraction (XRD) studies of single crystal [3] and powder [4] samples, although its vibrational spectrum was reported more than 20 years earlier [7]. The unit cell of $\epsilon-\mathrm{O}_{2}$ has $C 2 / m$ symmetry and contains four $\mathrm{O}_{2}$ molecules forming $\mathrm{O}_{8}$ units. Both $\alpha$ and $\delta-\mathrm{O}_{2}$ are antiferromagnetic and the magnetic collapse at the $\delta-\epsilon$ transition was predicted using molecular dynamics simulations 8 . This breakdown of the long-range antiferromagnetic order at about $8 \mathrm{GPa}$ was recently observed in a neutron scattering experiment [2]. Density functional theory (DFT) studies have found another chain-like structure to be energetically slightly more favorable than the $\mathrm{O}_{8}$ structure [9, 10, although it has not been observed in experiments.

Recent experiments [5] have shown that insulating $\epsilon-\mathrm{O}_{2}$ remains stable up to about $96 \mathrm{GPa}$ before undergoing a continuous displacive and isosymmetric transition to the $\zeta$ phase, in agreement with earlier predictions [10]. The metallic $\zeta$ phase [11, 12] has $C 2 / m$ symmetry [2] and superconducts at temperatures below $0.6 \mathrm{~K}$ [1]. Manybody perturbation theory $G W$ calculations [13, 14 have suggested that the metal-insulator transition occurs at lower pressures than the measured $\epsilon-\zeta$ transition pressure of $96 \mathrm{GPa}$. The above information, however, covers only a small part of the phase diagram and rather little is known about pure oxygen at pressures above $100 \mathrm{GPa}$.

A previous DFT study reported that oxygen molecules persist to at least $250 \mathrm{GPa}$ [10. It is interesting to speculate about the highest pressure to which oxygen molecules can survive, and whether oxygen forms polymeric materials as found in $\mathrm{N}_{2}$ [15, 17, $\mathrm{CO}$ [18, 19], and $\mathrm{CO}_{2}$ [20, 21], and predicted in $\mathrm{H}_{2}$ [22, 23]. Materials under terapascal pressures are of great interest in planetary science, for example, the pressure at the center of Jupiter is estimated to be about $7 \mathrm{TPa}$ 24]. Recent progress in dynamical shock wave 24 26 and ramped compression experiments [27, 28] has demonstrated that the terapascal pressure regime is becoming much more accessible.

The use of DFT computations combined with searching methods has provided a new route for predicting the structures and energetics of high-pressure phases [23, 29, 30]. A very recent study of phase transitions, melting, and chemical reactivity in $\mathrm{CO}_{2}$ found it to dissociate into carbon and oxygen above $33 \mathrm{GPa}$ and 1720 $\mathrm{K}$ [31, which adds further motivation for studying pure oxygen at high pressures. In this work we focus on oxygen at ultra-high pressures up to the terapascal regime, finding very surprising behavior.

We have used the $a b$ initio random structure searching (AIRSS) method [16, 22, 30, 32 and DFT calculations to identify low-enthalpy structures of oxygen in the multi TPa range. We used the Perdew-Burke-Ernzerhof (PBE) 33] generalized gradient approximation (GGA) exchange-correlation density functional. The searches were performed using the CASTEP plane-wave DFT code 34] and ultrasoft pseudopotentials. Searches were performed at selected combinations of $0.5,1,2,3,4,5$ and $8 \mathrm{TPa}$, with $6,8,9,10,12$ and 16 oxygen atoms per 
cell. A total of about 14000 relaxed structures were generated in the searches. The enthalpy-pressure relations were then recalculated using very hard projector augmented wave (PAW) pseudopotentials and the VASP code 35] with a plane-wave basis set cutoff energy of 900 $\mathrm{eV}$. Phonon and electron-phonon coupling calculations were performed using DFT perturbation theory and the ABINIT code [36], norm-conserving pseudopotentials, the PBE functional, and an energy cutoff of $1632 \mathrm{eV}$. Further details of the calculations are given in the supplementary material 37.

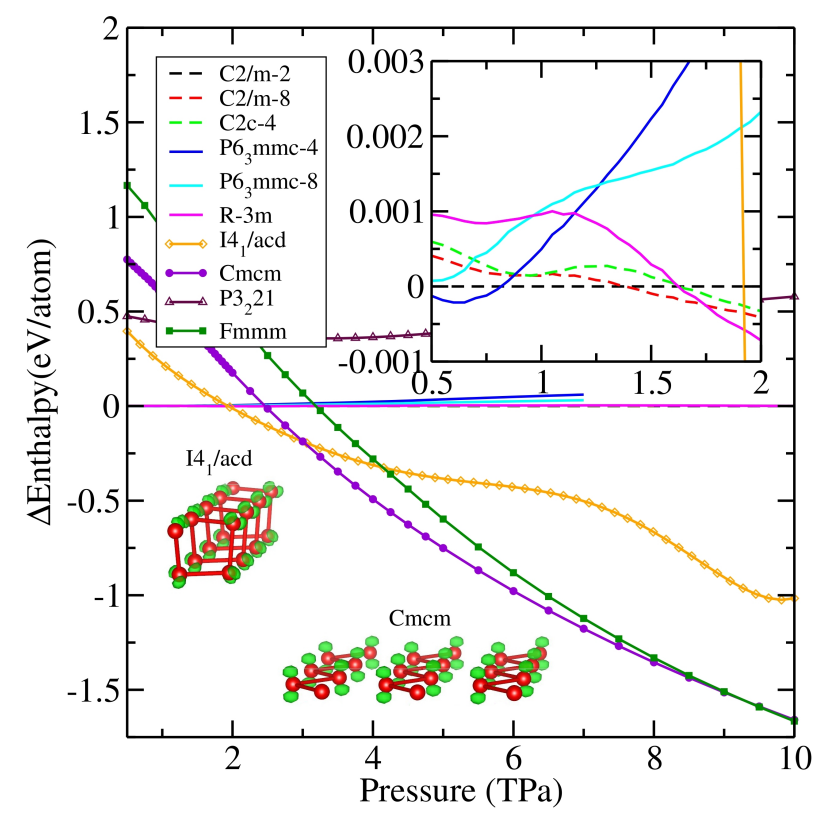

FIG. 1. Enthalpy-pressure relations for solid oxygen. The upper right inset shows the enthalpies of the molecular phases, while the insets in the lower left corner show the square spiral structure of $I 4_{1} / \mathrm{acd}$ and zig-zag chains of $\mathrm{Cmcm}$, respectively. The green bubbles represent the electron lone pairs.

The enthalpy-pressure relations for the most interesting structures are plotted in Fig. 1. We predict that at about 1.9 TPa molecular oxygen should transform into a square spiral-like structure belonging to space group $I 4_{1} /$ acd, as shown in the inset in the lower left corner of Fig. 1. Similar structures have been found in other heavier group VI elements, such as sulfur and selenium [38. The shortest $\mathrm{O}-\mathrm{O}$ bond length in $I 4_{1} /$ acd at $2 \mathrm{TPa}$ is about $1.15 \AA$, which is longer than that of the molecular phase at the same pressure (about $1.03 \AA$ ). The shortest O-O distance between the chains is about $1.55 \AA$, and the distance between the axes of neighboring square spirals is half of the lattice vector. The O-O-O angle in the helix is about $98.7^{\circ}$.

At about $3.0 \mathrm{TPa}$, the square-spiral phase transforms into a zig-zag chain-like phase of $\mathrm{Cmcm}$ symmetry, as shown in Fig. 1. The O-O bond length within the chains is $1.10 \AA$ at $3.5 \mathrm{TPa}$, while the inter-chain O-O separation in the plane is $1.33 \AA$. The O-O-O angle within the chain is $102.8^{\circ}$. With increasing pressure the chains in each layer of $\mathrm{Cmcm}$ gradually merge and at about $9.5 \mathrm{TPa}$ a structure of $F m m m$ symmetry forms in which each atom has four nearest neighbors at a separation of $1.05 \AA$.

The low-pressure molecular phases are very close in enthalpy, as the inset of Fig. 11 shows. Among these phases, $P 6_{3} / m m c-4, C 2 / m-2, C 2 / m-8$ and $R \overline{3} m$ become stable in turn on increasing the pressure from 0.5 to $1.9 \mathrm{TPa}$. More precise methods than DFT might be required to clarify the structural sequence as the enthalpy differences are so small. Although the $\mathrm{O}=\mathrm{O}$ bond in the $\mathrm{O}_{2}$ molecule is not as strong as the $\mathrm{N} \equiv \mathrm{N}$ and $\mathrm{C} \equiv \mathrm{O}$ triple bonds, $\mathrm{O}_{2}$ polymerizes at a much higher pressure than $\mathrm{N}_{2}(\sim 110$ $\mathrm{GPa}$ [15]) and CO (experiments show that $\mathrm{CO}$ polymerizes at about $5 \mathrm{GPa}[18$ while calculations predict that it could even polymerize at ambient pressure [19]). The fact that the molecular phases persist to pressures as high as $1.9 \mathrm{TPa}$ is intriguing. Electron counting arguments indicate that an oxygen atom can form two covalent bonds, either a double bond as in the $\mathrm{O}_{2}$ molecule or two single bonds, as would be expected in polymerized oxygen. The double bonds of the molecule are shorter and stronger than the single bonds of polymerized forms, and the lone electron pairs on the oxygen atoms result in bent bonds, as in the ozone $\mathrm{O}_{3}$ molecule, and give rise to strongly repulsive interactions between the molecules and between the polymeric chains. The great reluctance of oxygen to form more than two covalent bonds, the requirement that these bonds be bent, and the lone-pair repulsion greatly limits the forms of dense structures which may occur. The square spirals of the $I 4_{1} /$ acd structure are not well packed and, while the zig-zag chains of $\mathrm{Cmcm}$ pack more efficiently, there is no bonding between chains so that very dense structures are not formed. These factors lead to the persistence of molecular forms to high pressures, and the absence of stable framework structures up to at least $10 \mathrm{TPa}$.

We studied other possible candidate structural types such as buckled octagons of the type found in the S-I phase of sulfur $(F d d d)$ and the 3 -fold spiral-like $P 3_{2} 21$ structure of S-II [38, but these were found to be much less stable than the best structures obtained in our searches.

The electronic band structures and projected electronic densities of states (PDoS) of $R \overline{3} \mathrm{~m}$ at $1.8 \mathrm{TPa}$, $I 4_{1} /$ acd at $2.0 \mathrm{TPa}$ and $C m c m$ at $3.5 \mathrm{TPa}$ are shown in Fig. 22 decomposed into $s$ and $p$ components. As mentioned above, the enthalpies of the molecular phases are very close, but the $R \overline{3} m$ structure is the most likely molecular ground state prior to polymerization at 1.9 TPa. Our DFT calculations suggest that the $R \overline{3} m$ structure is metallic at $1.8 \mathrm{TPa}$ and the electronic density of states at the Fermi level mostly derives from the $p$ electrons.

Surprisingly, it turns out that the square spiral-like 

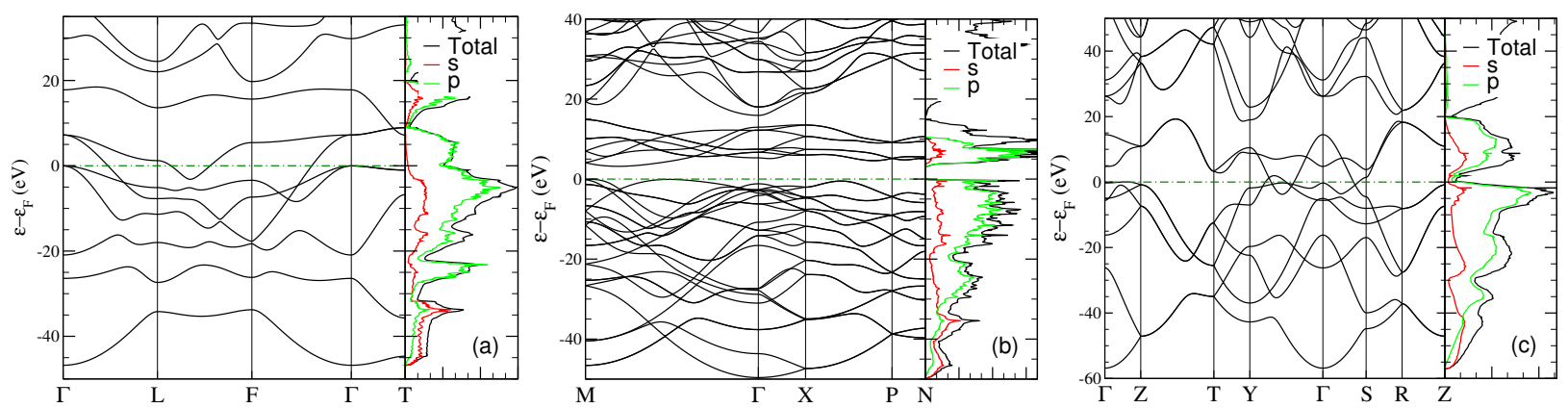

FIG. 2. Electronic band structures and projected densities of states (PDoS). (a) $R \overline{3} m$ at $1.8 \mathrm{TPa}$, (b) $I 4_{1} / a c d$ at $2.0 \mathrm{TPa}$, and (c) $\mathrm{Cmcm}$ at $3.5 \mathrm{TPa}$. The zero of energy is at the Fermi level.

a)
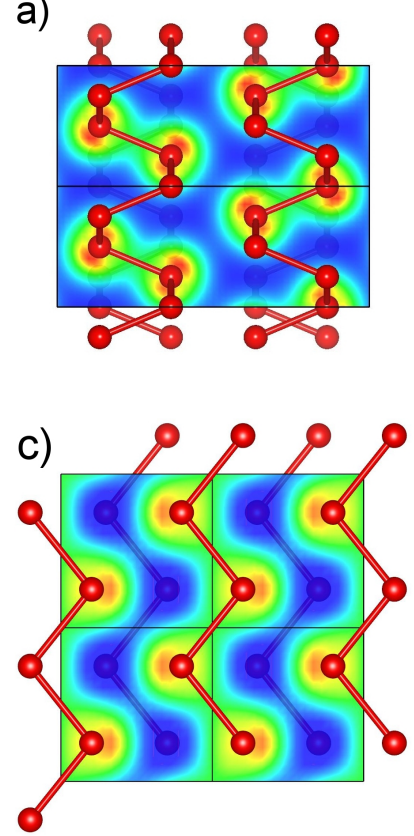

b)

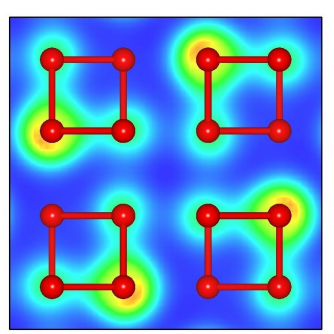

d)

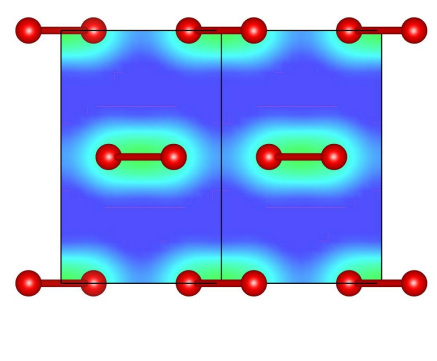

FIG. 3. Electronic densities of polymeric oxygen. (a) and (b) $I 4_{1} /$ acd at 2.0 TPa along the [100] and [001] directions, (c) and (d) Cmcm at 3.5 TPa along the [100] and [001] directions.

$I 4_{1} /$ acd phase has a band gap of $3.0 \mathrm{eV}$ at $2.0 \mathrm{TPa}$, as shown in Fig. 2(b). Considering that standard density functionals such as PBE are well-known for underestimating band gaps, $I 4_{1} /$ acd is expected to be a wide-gap semiconductor. As shown in the electron density plots of Fig. 3(a) and (b), and also in the charge density difference in the lower left inset of Fig. 1. the electrons are strongly localized. Strong covalent bonds are formed along the spiral chains and the projections of the electron density on the [100] and [001] planes show that the spirals are separated and not bonded to each other.

The calculated PDoS and band gap of the $\mathrm{Cmcm}$ phase (Fig. 2(c)) show that oxygen is metallic at 3.5 TPa. The

density of states at the Fermi level derives approximately equally from the $s$ and $p$ electrons and is smaller than that of the $R \overline{3} m$ structure (Fig. 2(a)). This indicates that the $\mathrm{Cmcm}$ phase is a weak metal and supports the conclusion that the lone pair electrons are not very extended. The electron density plots of Figs. 3(c) and (d) show that the zig-zag chains are not bonded to one another and that the layers are well separated.
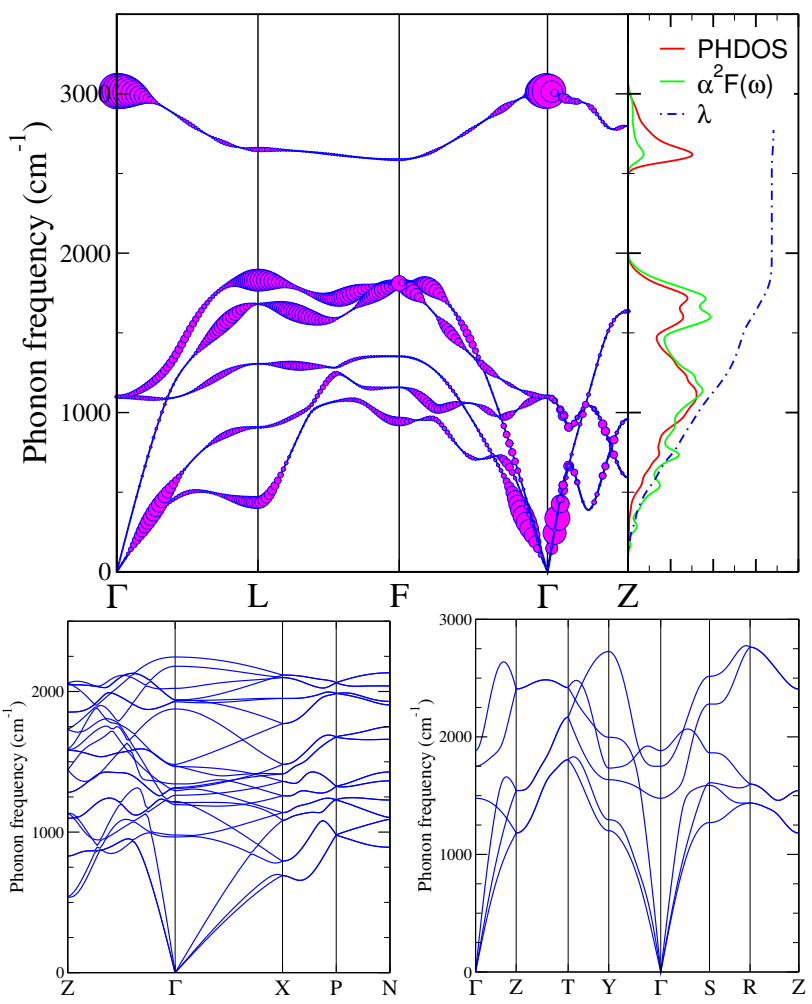

FIG. 4. Phonon dispersion relations of $R \overline{3} m$ at $1.8 \mathrm{TPa}$ (upper), $I 4_{1} /$ acd at $2.0 \mathrm{TPa}$ (lower left) and $C m c m$ at $3.5 \mathrm{TPa}$ (lower right). The blue circles with magenta filling show the phonon linewidth at different wave vectors.

We investigated the dynamical stability of the $R \overline{3} m$, $I 4_{1} /$ acd and $\mathrm{Cmcm}$ structures by calculating their 
phonon dispersion relations, as shown in Fig. 4. All of the structures are mechanically stable within certain pressure ranges. The phonons of both the molecular $R \overline{3} m$ and $C 2 / m$ phases (shown in supplementary [37]) are stable at 1.0 TPa, and $R \overline{3} \mathrm{~m}$ is also stable at $1.8 \mathrm{TPa}$. These structures have a group-subgroup relation which may indicate that oxygen molecules symmetrize upon increasing pressure near the transformation to polymeric phases. The molecular $\zeta$ oxygen is a superconductor at temperatures below $0.6 \mathrm{~K}$ [1]. We have therefore calculated the phonon linewidth, Eliashberg function $\alpha^{2} F(\omega)$, electron-phonon coupling (EPC) constant $\lambda$, and the logarithmic average phonon frequency $\omega_{l o g}$, to investigate whether the molecular $R \overline{3} \mathrm{~m}$ and polymeric $\mathrm{Cmcm}$ phases of oxygen are superconducting.

As shown in Fig. 4 (upper), the acoustic phonon modes and the intramolecular LO modes of $R \overline{3} m$ have relatively large linewidths close to the $\Gamma$ point, while the TO modes have large linewidths along $\mathrm{L}-\mathrm{F}$. We have used the Allen-Dynes modification of the McMillan equation for the superconducting transition temperature [39], $T_{c}=\omega_{l o g} / 1.2 \exp \left[-1.04(1+\lambda) / \lambda-\mu^{*}(1+0.62 \lambda)\right]$, where $\mu^{*}$ is the Coulomb pseudopotential, with typical values of $\mu^{*}$ between 0.10 and 0.13 . The calculated isotropic EPC constant for the $R \overline{3} m$ structure at $1.8 \mathrm{TPa}$ is about 0.34 and $T_{c}$ varies from 2.1 to $0.6 \mathrm{~K}$, which is close to the value observed for the $\zeta$ phase of about $0.6 \mathrm{~K}[1$.

The phonon band structure of $I 4_{1} /$ acd at $2.0 \mathrm{TPa}$ is shown in Fig. 4 (lower left). The phonon dispersion along $\mathrm{X}-\mathrm{P}$ and $\mathrm{P}-\mathrm{N}$ is small, indicating that the bonding between the square spirals is weak. The phonon dispersion relation of $\mathrm{Cmcm}$ at $3.5 \mathrm{TPa}$ (Fig. 4 lower right) shows the structure to be stable and highly anisotropic. Chainlike and layered structures also appear in other molecular systems, such as $\mathrm{CO}$ [19, 21] and $\mathrm{N}_{2}$ [17.

We have investigated oxygen under terapascal pressures using the AIRSS technique and ab initio DFT calculations. Oxygen molecules persist up to about 1.9 TPa, which is almost one order of magnitude larger than previously reported 10 and also much larger than other diatomic molecules such as $\mathrm{H}_{2}, \mathrm{~N}_{2}$ and CO. Oxygen polymerizes at about 1.9 TPa giving a square spiral-like structure of space group $I 4_{1} /$ acd, which is a semiconductor with a DFT band gap of about $3.0 \mathrm{eV}$. The $R \overline{3} \mathrm{~m}$ structure is superconducting at $1.8 \mathrm{TPa}$ and has a similar $T_{c}$ to the $\zeta$ phase. $R \overline{3} m$ is slightly more stable than other molecular forms at high pressures and its metallic oxygen molecules symmetrize before polymerization. At about 3.0 TPa, the system collapses to a denser metallic $\mathrm{Cmcm}$ phase, which consists of zig-zag chains. The stability of molecular and chain-like phases of highly-compressed solid oxygen demonstrates that the electron counting rule is effective up to multi-TPa pressures. Merging of the chains in each layer of $\mathrm{Cmcm}$ leads to the formation of a phase of $F m m m$ symmetry at $9.5 \mathrm{TPa}$ in which each atom has four nearest neighbors, which signals the even- tual failure of the electron counting rule to predict the bonding. The strongly repulsive interactions between the lone pairs hinders the polymerization of the molecules and the formation of more highly coordinated structures. The electronic structure of oxygen changes dramatically with increasing pressure. It transforms from the insulating $\epsilon$ phase to the molecular metal $\zeta$ phase, which persists up to $1.9 \mathrm{TPa}$, where it transforms into the semiconducting $I 4_{1} /$ acd polymeric phase, eventually returning to metallic behaviour with the appearance of the $\mathrm{Cmcm}$ phase at about 3.0 TPa.

J.S. gratefully acknowledges financial support from the Alexander von Humboldt (AvH) foundation. C.J.P. and R.J.N. were supported by the EPSRC. The calculations were carried out at BovILAB@RUB (Bochum), on the supercomputers at NRC (Ottawa) and UCL.

* jian.sun@theochem.rub.de

[1] K. Shimizu et al., Nature 393, 767 (1998).

[2] I. Goncharenko, Phys. Rev. Lett. 94, 205701 (2005).

[3] L. F. Lundegaard et al., Nature 443, 201 (2006).

[4] H. Fujihisa et al., Phys. Rev. Lett. 97, 085503 (2006).

[5] G. Weck, S. Desgreniers, P. Loubeyre, and M. Mezouar, Phys. Rev. Lett. 102, 255503 (2009).

[6] C. Barrett, L. Meyer, and J. Wasserman, J. Chem. Phys. 47, 592 (1967).

[7] M. Nicol, K. R. Hirsch, and W. B. Holzapfel, Chem. Phys. Lett. 68, 49 (1979).

[8] S. Serra, G. Chiarotti, S. Scandolo, and E. Tosatti, Phys. Rev. Lett. 80, 5160 (1998).

[9] J. B. Neaton and N. W. Ashcroft, Phys. Rev. Lett. 88, 205503 (2002).

[10] Y. M. Ma, A. R. Oganov, and C. W. Glass, Phys. Rev. B 76, 064101 (2007).

[11] S. Desgreniers, Y. Vohra, and A. Ruoff, J. Phys. Chem. 94, 1117 (1990).

[12] Y. Akahama et al., Phys. Rev. Lett. 74, 4690 (1995).

[13] D. Y. Kim et al., Phys. Rev. B 77, 092104 (2008).

[14] J. S. Tse et al., Solid State Communications 145, 5 (2008).

[15] M. I. Eremets et al., Nature Mat. 3, 558 (2004).

[16] C. J. Pickard and R. J. Needs, Phys. Rev. Lett. 102, 125702 (2009).

[17] Y. M. Ma et al., Phys. Rev. Lett. 102, 065501 (2009).

[18] M. J. Lipp, W. J. Evans, B. J. Baer, and C. S. Yoo, Nature Mat. 4, 211 (2005).

[19] J. Sun, D. D. Klug, C. J. Pickard, and R. J. Needs, Phys. Rev. Lett. 106, 145502 (2011).

[20] V. Iota et al., Nature Mat. 6, 34 (2007).

[21] J. Sun et al., Proc. Natl. Acad. Sci. U.S.A. 106, 6077 (2009).

[22] C. J. Pickard and R. J. Needs, Nature Phys. 3, 473 (2007).

[23] J. M. McMahon and D. M. Ceperley, Phys. Rev. Lett. 106, 165302 (2011).

[24] R. Jeanloz et al., Proc. Natl. Acad. Sci. U.S.A. 104, 9172 (2007).

[25] M. D. Knudson, M. P. Desjarlais, and D. H. Dolan, Sci- 
ence 322, 1822 (2008).

[26] J. H. Eggert et al., Nature Phys. 6, 40 (2010).

[27] J. Hawreliak et al., Astrophys. Space Sci. 307, 285 (2007).

[28] D. K. Bradley et al., Phys. Rev. Lett. 102, 075503 (2009).

[29] J. Sun, D. D. Klug, and R. Martonak, J. Chem. Phys. 130, 194512 (2009).

[30] C. J. Pickard and R. J. Needs, Nature Materials 9, 624 (2010).

[31] K. D. Litasov, A. F. Goncharov, and R. J. Hemley, Earth Planet. Sci. Lett. 309, 318 (2011).

[32] C. J. Pickard and R. J. Needs, J. Phys.: Condens. Matter
23, 053201 (2011).

[33] J. P. Perdew, K. Burke, and M. Ernzerhof, Phys. Rev. Lett. 77, 3865 (1996).

[34] S. J. Clark et al., Z. Kristallogr. 220, 567 (2005).

[35] G. Kresse and J. Furthmüller, Comp. Mat. Sci. 6, 15 (1996).

[36] X. Gonze et al., Comp. Mat. Sci. 25, 478 (2002).

[37] See EPAPS Document No. xxxxxxxxxx. For more information on EPAPS, see http://www.aip.org/pubservs/epaps.html

[38] O. Degtyareva et al., Nature Mat. 4, 152 (2005).

[39] P. B. Allen and R. C. Dynes, Phys. Rev. B 12, 905 (1975). 